\title{
Advanced CAD/CAM Techniques for 5-Axis Machining of Free-Form Surfaces
}

Nguyen Van Tuong

Faculty of Mechanical Engineering, Nha Trang University. 02 Nguyen Dinh Chieu, Nha Trang, Khanh Hoa, Viet Nam. E-mail: tuongnv@ntu.edu.vn

This paper presents some advanced techniques on CAD modelling and CAM programming for 5-axis machining of free-form surfaces. In the CAD stage, based on surface partitioning, the design surface can be created with separate regions such as convex, concave and saddle. Point-based techniques are used to create the original surface and the boundary curves of the regions. Some other CAD/CAM techniques for determining tool sizes and tool orientations are also proposed to generate gouge-free tool paths for each region. A simple B-spline surface was given as an example to demonstrate the proposed techniques implemented in Creo Parametric. The points on the design surface and on the boundaries were generated by a Matlab program developed by the author.

Keywords: Surface partitioning, Free-form surface, 5-axis machining, Gouge-free tool path.

\section{Introduction}

Nowadays, free-form surfaces have become the reality of series production in aviation, automotive, and consumer goods industries when CAD systems and modern scanning instruments entered the re-engineering process [5]. These kinds of surfaces can be machined on 3-axis computer numerical control (CNC) machines by using ball-end cutters or 5- axis CNC machines by using ballend, toroidal or flat-end cutters. Irrespective of whether maching is done in 3-axis or 5-axis mode, the productivity will be low if only one cutting tool is used to machine the entire surface. This is because of the limitation of the tool diameter that depends on the radius of the design surface. When choosing a cutting tool for a particular surface, the tool diameter is restricted to a determined value that must not cause local gouging when the tool is machining in concave and/or saddle regions. To improve the machining efficiency, free-form surfaces can be machined region by region with different tools $[2,6,15]$. By so doing, the concave regions are often milled by smaller ball-end cutters and the other regions can be finished by bigger ball-end cutters or by flat-end cutters.

In general, a free-form surface has regions such as convex, concave, plane and saddle [6, 7]. In CAD/CAM (Computer-Aided Design/Computer-Aided Manufacturing) packages, users can not separately choose every region in the CAM programming stage, so that the design surface should be partitioned into regions in the modeling stage. Every CAD system has a particular method to create free-form surfaces. However, in current CAD systems, it is imposible to create free-form surfaces with separate regions unless special techniques are used. $[4,11$, 12]

Free-form surface partitioning has been studied by various researchers using different approaches. To create the CAD model of the partitioned surfaces, some researchers used $\mathrm{C}++$ to develop their own application programs and run them in Open GL environment [1, 8-10]. We introduced a practical approach for surface partitioning based on surface curvatures and chain codes techniques [15]. In this research, a Matlab program was developed for calculating purposes. This program is available for B-spline surfaces. The input of the program is the control net and knot vectors and the outputs are points with their coordinates on the design surface and on the boundaries as well. This method is very universally because once the points are established, the design surface and the boundary curves can be created easily by many CAD or $\mathrm{CAD} / \mathrm{CAM}$ packages. In this paper, some advanced $\mathrm{CAD} / \mathrm{CAM}$ techniques for modelling and tool path planning free-form surface machining are presented. These techniques were implemented in Creo Parametric (PTC, USA).

\section{Building cad models of free-form surfaces}

Free-form surfaces can be designed directly by using $\mathrm{CAD}$ or $\mathrm{CAD} / \mathrm{CAM}$ packages or obtained through surface fitting from a digitized data set. In the latter case, often used in reverse engineering, a scanner digitizes surfaces of an existing object to get a set of points and feeds them into a mathematical model for surface fitting. In the former case, designers have to move control points on the screen to get the desired surface.

Generally, free-form surfaces can be represented in the forms of Bezier surface, B-spline surface, or Non-uniform Rational B-spline surface (NURBS). Because Creo Parametric does not support directly creating these kinds of surfaces from their control points, a replacement technique is required. In this research, ibl files (files with the extension .ibl) were used to creat B-spline surfaces from imported datum curves. In fact, datum curves were created in the form of an ibl file and this file was then imported into Creo Parametric to create a B-spline surface. It should be noted that the datum curve is a spline created from more than two points [13] and the B-spline surface goes through these curves to be created.

Tab. 1 Structure of the ibl file format

\begin{tabular}{|c|}
\hline OpenArclength \\
\hline Begin section ! 1 \\
\hline Begin curve ! 1 \\
\hline Points list \\
\hline Begin section ! 2 \\
\hline Begin curve ! 1 \\
\hline Points list \\
\hline$\ldots$ \\
\hline
\end{tabular}


An ibl file is a text file that contains geometry defined by three-dimensional (3D) points. The structure of the ibl file format is in the Tab. 1.

In the ibl file format, there is a list of points for each curve. These points are represented with $\mathrm{x}, \mathrm{y}$ and $\mathrm{z}$ coordinates. Actually, they are datum points to which Creo Parametric will fit splines. The points for creating curves in the ibl file can be received by sampling points on the design surface from the Matlab program.

In order to create the original CAD model of a freeform surface in Creo Parametric, first an ibl file should be created by Notepad or WordPad applications. Once the ibl file has been created, the design surface can be created by using the Blend from file command to import curves from an existing ibl file.

To split the original surface into regions, the boundary curves of partitioned regions are required. These 3D curves can be created from the boundary points whose coordinates are obtained from the calculating results in the Matlab program as mentioned before.

In Creo Parametric, to create a 3D curve through datum points, the Offset Coordinate System Datum Point Tool can be used. In this tool, the coordinates of the points should be stored in files with the extension .pts and they are imported form the Import tab in the Offset CSys Datum Point window. Once the 3D points have been established on the boundaries, the Insert a Datum Curve tool can be used to get a spline curve through those points.

Creo Parametric supplies the Surface Trim tool in the Style tool to divide the design surface into regions. In this case, users should choose 3D curves on the surface as trim factors. The $3 \mathrm{D}$ curves mentioned in the paragraph above will be used for the trimming.

\section{Generating free-gouge tool paths}

Local and global gouging is one of the most critical problems in free-form surface machining. Local gouging refers to the removal of excess material in the vicinity of the cutter contact point as the tool moves along the tool path. At every cutter contact point, local gouging occurs when the radius of the local surface curvature is smaller than that of the cutter. Global gouging is defined as interference between the tool body and the design surface.

In this study, by surface partitioning, the design surface is partitioned into regions. Thanks to the effectiveness of surface partitioning, gouge detection and correction can be effectively performed in CAD/CAM packages in two forms as follows: (1) defining suitable tools for machining the regions under the condition of local gouge-free, and (2) choosing reasonable orientation of the tool when machining each region and detecting global gouging.

In general, ball-end cutters are often used for finishing free-form surfaces. The diameter of a ball-end cutter is restricted by local gouging conditions when machining concave and saddle regions. A ball-end cutter will not cause any local gouges on the design surface if the cutter radius is smaller than the smallest radius of the selected region. In Creo Parametric, users can perform an analysis of surface radius to display the minimum radii for a surface [13]. The possible maximum radius of the cutter can be defined from the minimum radius of each region.

Compared with 3 -axis milling, 5 -axis milling is more complicated due to the two additional degrees of freedom. In 3-axis milling technology, a tool was used without tilting the tool axis, while in 5-axis milling technology, a milling tool was used with an angle in the feed direction [14]. The major difference between these two operations is the existence of the lead and tilt angles. The lead angle is the rotation of the tool axis about the cross-feed axis, whereas the tilt angle is the rotation about the feed axis with respect to the surface normal [3]. In practice, the lead and tilt angles are often selected by trial and error and based on the experience of users. In Creo Parametric, these angles are specified by parameters LEAD_ANGLE and TILT_ANGLE [13]. However, sometimes, this method cannot be used because of global gouging, e.g. machining deep concave regions. In this case, a strategy with variable angles between the tool axis and normal vector must be applied. The following are some techniques which can satisfy that requirement: (i) The tool axis always goes through a curve and (ii) The tool axis always goes through a specified point. These suggested techniques can be very effective to avoid gouging on the partitioned surfaces.

\section{Application}

A number of examples have been implemented in our research. In this paper, a simple case is presented for the purpose of demonstration.

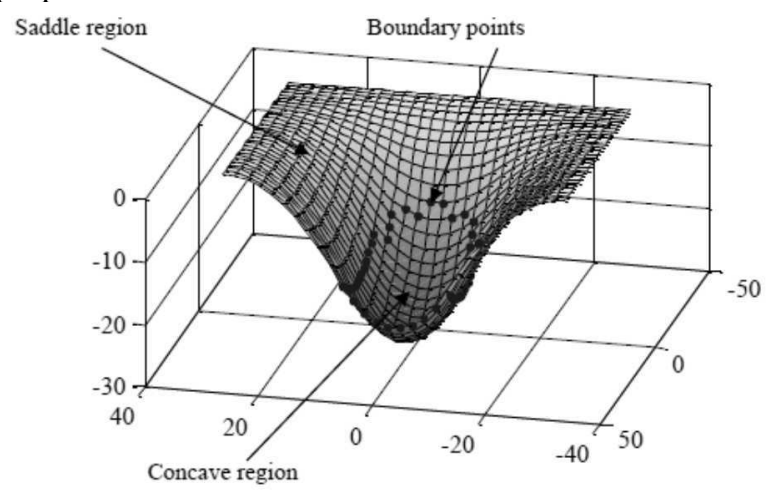

Fig. 1 A B-spline surface constructed in Matlab

Assume a B-spline surface defined by $5 \times 5$ control net as in Tab. 2. The uniform knot vector in both parametric directions is [ $\left[\begin{array}{llllllll}0 & 1 & 2 & 3 & 4 & 5 & 6 & 7\end{array}\right]$.

Tab. 2 Control net of a B-spline surface

\begin{tabular}{|c|c|c|c|c|}
\hline$(-40,-40,0)$ & $(-20,-40,0)$ & $(0,-40,0)$ & $(20,-40,0)$ & $(40,-40,0)$ \\
$(-40,-20,0)$ & $(-20,-20,0)$ & $(0,-20,0)$ & $(20,-20,0)$ & $(40,-20,0)$ \\
$(-40,0,0)$ & $(-20,0,0)$ & $(0,0,-30)$ & $(20,0,0)$ & $(40,0,0)$ \\
$(-40,20,0)$ & $(-20,20,0)$ & $(0,20,-40)$ & $(20,20,0)$ & $(40,20,0)$ \\
$(-40,40,0)$ & $(-20,40,0)$ & $(0,40,-20)$ & $20,40,0)$ & $40,40,0)$ \\
\hline
\end{tabular}


By executing the Matlab program developed in this research, the points on the design surface and the points on the boundaries were obtained. Figure 1 shows a Matlab figure of the design surface which was sampled to get a set of 900 points (for a better view). According to the calculated results, there is one concave region and one convex region on this surface. Therefore, one boundary is required to split the design surface into 2 regions. In the research, the design surface was sampled to get a set of 4 900 points. In this case, there were 125 points on the bounday between the two regions.

An ibl file with 15 curves was created for this example. Each curve has 15 points. Tab. 3 shows the list of points of the first and the last curve.

Tab. 3 Example of list points of an ibl file

\begin{tabular}{|llll}
\hline \multicolumn{4}{l}{ OpenIndexArclength } \\
Begin section ! 1 \\
Begin curve ! 1 \\
1 & -30 & -30 & 0 \\
2 & -25.71428571 & -30 & 0 \\
3 & -21.42857143 & -30 & 0 \\
4 & -17.14285714 & -30 & 0 \\
5 & -12.85714286 & -30 & 0 \\
6 & -8.571428571 & -30 & 0 \\
7 & -4.285714286 & -30 & 0 \\
8 & 0 & -30 & 0 \\
9 & 4.285714286 & -30 & 0 \\
10 & 8.571428571 & -30 & 0 \\
11 & 12.85714286 & -30 & 0 \\
12 & 17.14285714 & -30 & 0 \\
13 & 21.42857143 & -30 & 0 \\
14 & 25.71428571 & -30 & 0 \\
15 & 30 & -30 & 0
\end{tabular}

Begin section! 15

Begin curve ! 1
$1-30$
$30 \quad 0$
$2-25.71428571 \quad 30 \quad-0.68877551$
$3 \quad-21.42857143 \quad 30 \quad-2.755102041$
$\begin{array}{lllll}4 & -17.14285714 & 30 & -6.198979592\end{array}$
$\begin{array}{lllll}5 & -12.85714286 & 30 & -11.02040816\end{array}$
$\begin{array}{lllll}6 & -8.571428571 & 30 & -16.98979592\end{array}$
$\begin{array}{lllll}7 & -4.285714286 & 30 & -21.12244898\end{array}$
$\begin{array}{llll}8 & 0 & 30 & -22.5\end{array}$
$9 \quad 4.285714286 \quad 30 \quad-21.12244898$
$\begin{array}{lllll}10 & 8.571428571 & 30 & -16.98979592\end{array}$
$\begin{array}{lllll}11 & 12.85714286 & 30 & -11.02040816\end{array}$
$\begin{array}{lllll}12 & 17.14285714 & 30 & -6.198979592\end{array}$
$\begin{array}{lllll}13 & 21.42857143 & 30 & -2.755102041\end{array}$
$\begin{array}{lllll}14 & 25.71428571 & 30 & -0.68877551\end{array}$
$\begin{array}{lll}1530 & 30 \quad 0\end{array}$

In Creo Parametric, by importing curves from the ibl file, the B-spline surface was created as shown in Figure 2.
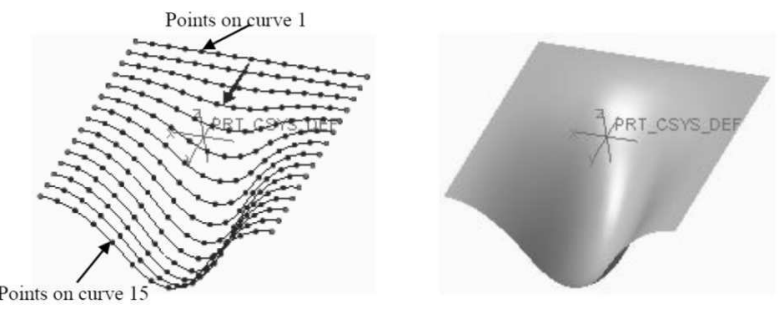

Fig. 2 The curves from the ibl file and the B-spline surface

Figure 3 below shows the CAD model of the design surface after three main steps of modelling. The original surface was created from an ibl file with 15 curves. Each curve has 15 points.
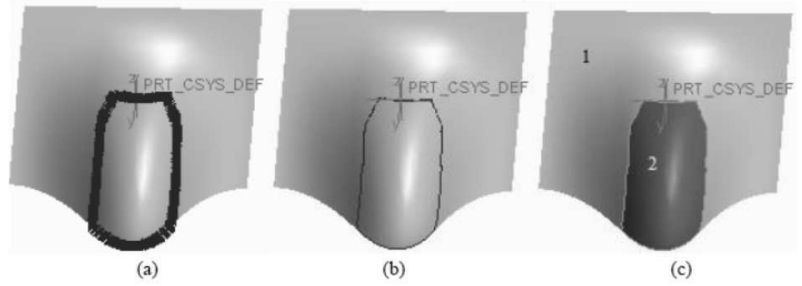

Fig. 3 CAD model of the design surface: (a) original surface with boundary points, (b) original surface with boundary curve, and (c) partitioned surface: 1- saddle region, 2-concave region

The minimum radii for the concave and saddle regions are $4.6575 \mathrm{~mm}$ and $12.6627 \mathrm{~mm}$, respectively. An example of the minimum radius of the concave region is shown in Figure 4.

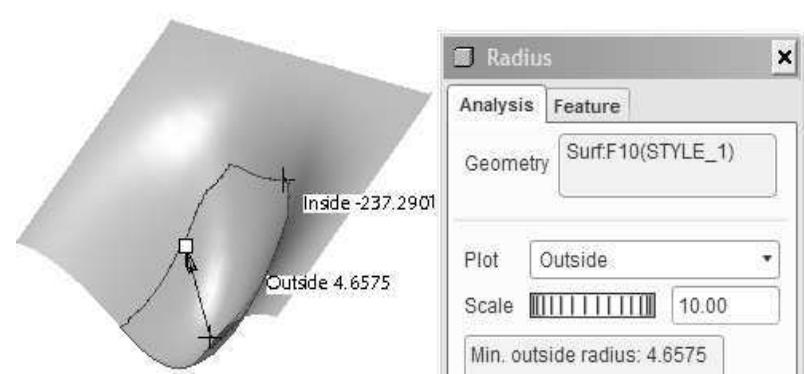

Fig. 4 The minimum radius of the concave region

One of the objectives of machining free-form surfaces region by region is to improve machining productivity. This means that the proposed method (with partitioned surface) is really effective when the machining distance is shorter than that of the conventional method (with unpartitioned surface). For comparison, some constraints should be applied for both methods. Regardless of which tool is used for machining, the values of scallop height and tolerance for finishing should be the same.

Scallops are ridges, cusps and other surface protrusions left between adjacent overlapping tool passes that extend above the design surface profile. In Creo Parametric, the SCALLOP_HGT parameter is used to control tool step by specifying the maximum allowable scallop for surface milling [13]. The tool path approximates curved geometry by a series of straight line segments. The maximum distance that the straight line path deviates from the curved geometry is set by the TOLERANCE parameter in Creo Parametric [13].

The two regions of the design surface can be finished by ball-end cutters. From the values of the maximum curvatures of the two regions, to avoid local gouging the radii of the cutters must be smaller than $4.6575 \mathrm{~mm}$ and 
$12.6627 \mathrm{~mm}$ to machine the concave and saddle regions, respectively. In this application, after a rough cut the whole surface in 3-axis mode by a flat-end mill of $10 \mathrm{~mm}$ in diameter, the machining strategy for the partitioned surface can be as follows:

- Finishing cut in 5-axis mode for the saddle region by a $20 \mathrm{~mm}$ four-flute ball-end cutter,

- Finishing cut in 5-axis mode for the concave region by a $8 \mathrm{~mm}$ two-flute ball-end cutter.
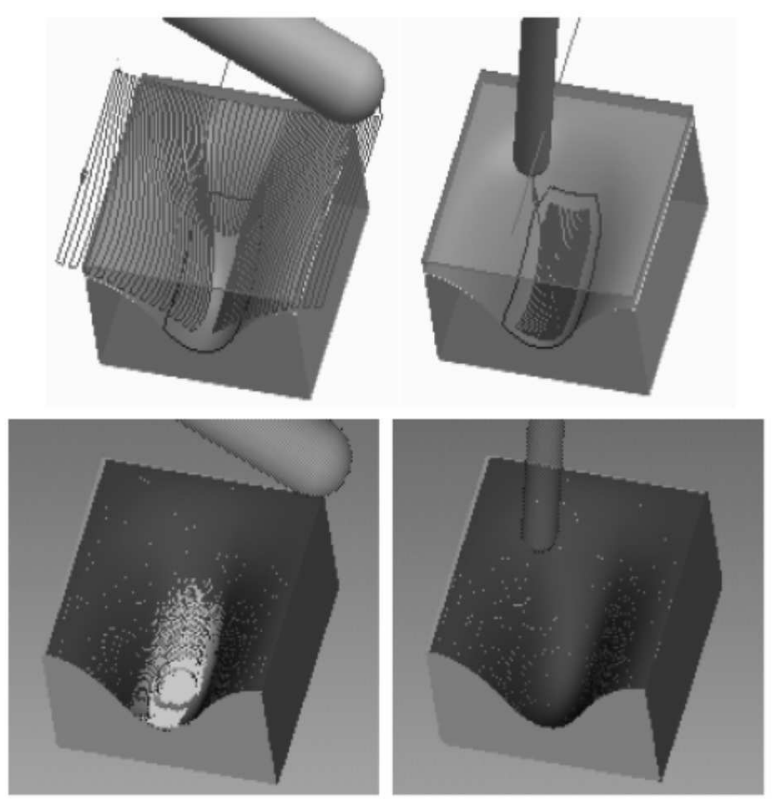

Fig. 5 Tool paths for each region and machining simulation results

The design surface has a symmetrical shape. The concave region is rather deep and the areas near the boundary on both regions are quite steep. In consequence, fixed lead and tilt angles are not available for this application. This is because the tool will cause global gouges on the other side of the saddle region when it is machining this region. Gouges also occur on the saddle region when the tool is machining on the concave region. To create gouge-free tool paths, the techniques in which the tool axis always goes through a fixed curve or point was used. Here, the tools were set to go through a straight line located at $30 \mathrm{~mm}$ above the top of the design surface.

To compare the machining distance of the finish cut between the proposed method (partitioned surface) and the traditional method (un-partitioned surface), the tolerance and scallop height must be the same for all tools. Here, the SCALLOP_HGT and TOLERANCE parameters were set to $0.01 \mathrm{~mm}$ and $0.02 \mathrm{~mm}$ respectively. Figure 5 shows the tool paths generated for each region and the machining simulation results without gouges.

The machining distance for the un-partitioned surface and the partitioned surface estimated by Creo Parametric were $7474.736 \mathrm{~mm}$ and $5594.084 \mathrm{~mm}$ respectively. It can be seen that the machining distance of the partitioned surface is much shorter than that of the un-partitioned surface. The total length of the tool paths generated by the proposed method is approximately equal to $75 \%$ of the traditional method. This reduction comes from the tool paths for the saddle region having a much shorter distance. This is because the saddle region was programed to be cut by a bigger tool diameter while the tolerance and scallop height were also maintained. Assuming that the cutting speed was $50 \mathrm{~m} / \mathrm{min}$, the feed rate per tooth is $0.1 \mathrm{~mm} / \mathrm{rev} /$ tooth. Then, the machining time estimated by Creo Parametric for the traditional method and proposed method were 18.781 mimutes and $16.699 \mathrm{mi}-$ mutes respectively. This means that the proposed method can save up to $11,1 \%$ of the machining time. If the cutting speed is incresed by $50 \%$ when machining the saddle region, the machining time of the whole surface is 12.297 minutes. In this case, a reduction of $34.5 \%$ of the machining time can be made. Hence, it can be said that the proposed method is really effective in terms of machining distance and machining time, compared to the traditional method. Figure 7 shows the result of the machining sequences of the design surface on the Integrex 100-IV (Mazak, Japan).

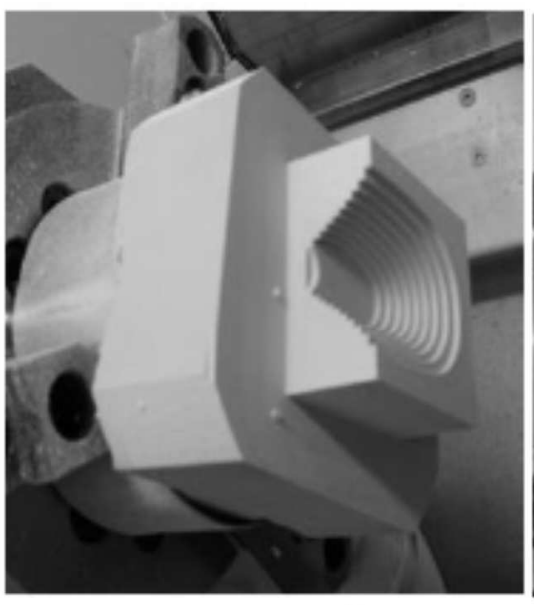

a)

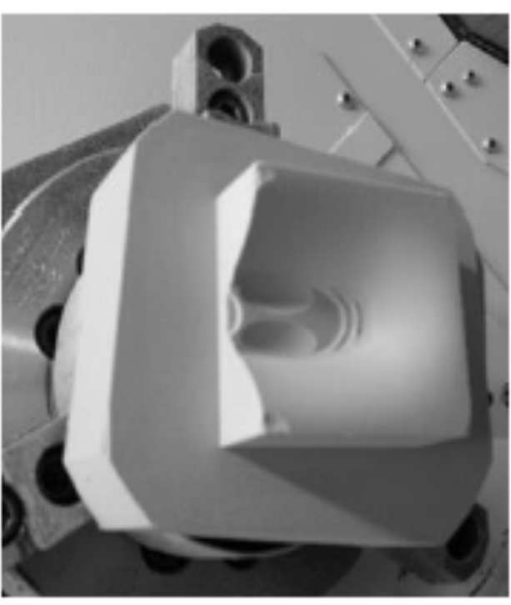

b)

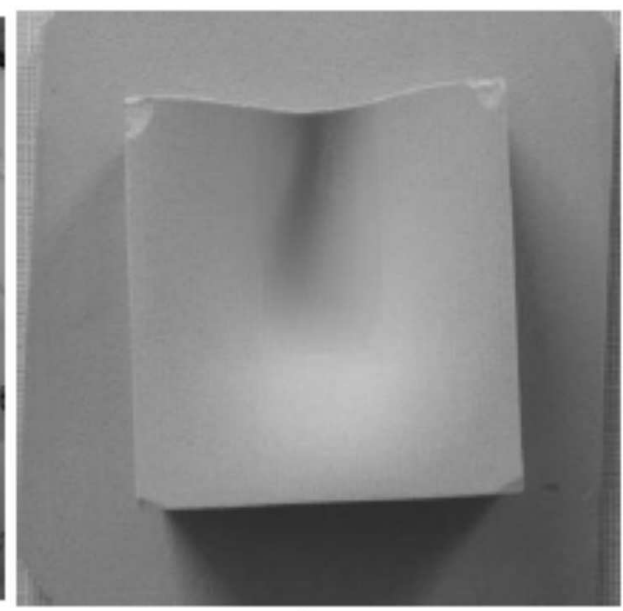

c)

Fig. 7 Machining sequences of the design surface: (a) part after the roughing cut; (b) part after the finishing cut for the saddle region; (c) final part 


\section{Conclusion}

The proposed CAD/CAM techniques can be useful for modelling and tool path planning for free-form surfaces. From points archieved from a Matlab program, a free-form surface and the boundary curves which can define convex, concave and saddle regions of the design surfaces can be created in Creo Parametric. The boundary curves can be used as a trim tool to partition the design surface regions in the CAD modelling stage. In the CAD stage, the gouge-free tool paths for each region can easily be created with different tools and therefore the machining distance and the machining time can be reduced considerably. Applying the proposed CAD techniques for modelling free-form surfaces using a digitized data set is the future work of this study.

\section{Acknowledgement}

The work upon which this paper is based is a part of MPO FI-IM5/065 research program grant at the Technical University of Liberec, Czech Republic.

\section{References}

[1] BEY, M., BENDIFALLAH, M., KADER, S., BOUKHALFA, K. (2008). Cutting tool combination and machining strategy affectation based on the determination of local shapes for free form surfaces. In: Proceeding of the International Conference on Smart Manufacturing Application, pp. 120-125.

[2] BILEK, O., ROKYTA, L., SIMONIK, J. (2012). CAM in the Production of Casting Patterns. In: Manufacturing Technology, Vol. 12, No 12, pp. 712.

[3] BUDAK, E., OZTURK, E.,TUNC, L., T. (2009). Modelling and simulation of 5-axis milling processes. In: CIRP Annals - Manufacturing Technology, Vol. 58, pp. 347-350.

[4] CUBONOVA, N. (2013). Postprocessing of CL Data in CAD/CAM system Edgecam using the Constructor of postprocessors. In Manufacturing Technology. Vo. 13, No 13, pp. 158-164.

[5] DOVICA, M., BUSA J., SPISAK, E., FABIAN, M., IZOL, P., FABIANOVA, J. (2016) Assessment of complex free form surfaces with surface profile deviation. In Manufacturing Technology, August 2016, Vol. 16, No. 4, pp. 673-680.
[6] ELBER, G., COHEN, E. (1993). Second order surface analysis using hybrid symbolic and numeric operators. In: Transactions on Graphics, Vol. 12, pp. 160-178.

[7] FABIAN, M., STANOVA, E., FEDORKO, G., KMET, S., FABIANOVA, J., KRAJNAK, J. Parametric CAD model of a double-lay six strand wire rope. In Manufacturing Technology, Vol. 16, No 3, pp. 489-496.

[8] LI, L., L., ZHANG, Y., F. (2004). Cutter selection for 5-axis milling based on surface decomposition. In: Proceeding of the 8th International Conference on Control, Automation, Robotics and Vision, Vol. 3, pp. 1863- 1868.

[9] LI, L., L., ZHANG, Y.,F. (2005). Flat-end cutter accessibility determination in 5-axis milling of sculptured surfaces. In: Computer-Aided Design \& Applications, Vol. 2, pp. 203-212.

[10] LI, L., L., ZHANG, Y.,F. (2006). An integrated approach towards process planning for 5-axis milling of sculptured surfaces based on cutter accessibility map. In: Computer-Aided Design \& Applications, Vol. 3, pp. 249-258.

[11] NAPRSTKOVA, N. (2011) Using of catia V5 software for teaching at faculty of production technology and management, 10th International Scientific Conference on Engineering for Rural Development, Jelgava, Latvia, pp. 554-557.

[12] NAPRSTKOVAN, N. (2010) Students Connecting to Production Problems Resolutions in CAD/CAM Area. In: 9th International Scientific Conference on Engineering for Rural Development, Jelgava, Latvia, pp. 310-314.

[13] PARAMETRIC TECHNOLOGY CORPORATION, Creo Parametric Help Center, http://support.ptc.com/help/creo/creo_pma/usascii/index.html.

[14] SADILEK,M., KOUSAL, L., NAPRSTKOVA, N., SZOTKOWSKI, T., HAJNYS, J. (2018). The analysis of accuracy of machined surfaces and surfaces roughness after 3axis and 5axis milling. In Manufacturing Technology, Vol. 18, No 6, pp. 1015-1022.

[15] TUONG, N.,V., POKORNY, P. (2010). A practical approach for partitioning free-form surfaces. In: International Journal of Computer Integrated Manufacturing, Vol. 23, pp. 992-1001. 